(2) Open Access Full Text Article

REVIEW

\title{
Assessing and optimizing health-related quality of life during and after cancer treatment in adolescents and young adults
}

This article was published in the following Dove Press journal:

Clinical Oncology in Adolescents and Young Adults

12 December 2014

Number of times this article has been viewed

\author{
Dava Szalda' \\ Esther Kim² \\ Jill P Ginsberg ${ }^{1,2}$ \\ 'Division of Oncology, The Children's \\ Hospital of Philadelphia, ${ }^{2}$ Perelman \\ School of Medicine at the University \\ of Pennsylvania, Philadelphia, PA, USA
}

Correspondence: Dava Szalda Colket Translational Research Building 10-300-04, The Children's Hospital of Philadelphia, 350I Civic Center Blvd, Philadelphia, I9I04 PA, USA

Email szaldad@email.chop.edu
Abstract: Adolescent and young adults (AYAs) with cancer have needs unique from their pediatric or adult counterparts at diagnosis, during treatment, and throughout survivorship care. Healthcare teams may find it difficult to assess and manage the complex psychosocial needs of AYA patients, however, attention to the multi-faceted care of the AYA oncology patient may directly affect health outcomes and quality of life. Comprehensive AYA care therefore must be maximized during treatment and in transition to survivorship care by including assessments of general health, sexual health, mental health, health behaviors, and conversations concerning transition to survivorship and adulthood with AYA patients. Identifying and treating issues that arise in the AYA population may help promote adherence to treatment, engagement in follow-up care, and ultimately quality of life for this unique group of patients.

Keywords: quality of life, sexual health, health behaviors, mental health, transition, survivorship

\section{Introduction}

Although the health outcomes of children and adults with cancer have received much attention, adolescents and young adults are now recognized as a distinct group because of their cancer biology and unique psychosocial issues. ${ }^{1}$ The National Cancer Institute defines the Adolescent and young adult (AYA) population as those between ages 15 and 39 years. ${ }^{2}$

Each year, approximately 70,000 AYAs are diagnosed with cancer. ${ }^{1,2}$ With improvements in treatment and survival, the majority of those diagnosed with cancer as an adolescent or young adult will become long-term survivors, but these improvements in survival may come at some cost, affecting level of functioning and overall quality of life.

Quality of life is a multidimensional concept that comprises elements of physical, social, and psychological health, as well as the patients' perceived health status and well-being, all of which are affected and may be disrupted during cancer diagnosis and treatment. ${ }^{3}$ As adolescence and young adulthood is already a time when personal experimentation, development of new relationships, exploring sexual awareness, and exposure to or engagement in risk-taking behavior are all considered essential developmental tasks, AYA oncology patients face a variety of disease- and treatmentrelated health and psychosocial issues that may adversely affect their quality of life, in addition to issues that are common to normal growth and development.

AYAs also may face more transitions during oncologic care than their pediatric or adult counterparts. During diagnosis and treatment, AYAs fall in a position between pediatric and adult healthcare service models, neither of which may be appropriate 
to meet their healthcare and psychosocial needs. AYAs will also transfer from receiving therapy to survivorship care. ${ }^{4}$ The unique psychological effect from cancer diagnosis and treatment often continues to affect outcomes well into adulthood and as survivors.

For these reasons, the specific needs of AYA cancer patients and cancer survivors need to be addressed. There is support and initiative for high-quality AYA cancer care at national levels. The Institute of Medicine and National Cancer Institute have identified areas in need of improvement in terms of both health outcomes and quality of life for this group. ${ }^{2,5}$ This article reviews issues that should be addressed during appropriate age-based cancer care for the AYA population to optimize quality of life during and after cancer treatment, including general health, sexual health, mental health, health behaviors, and transition to survivorship and adulthood.

\section{General health}

\section{Promotion of a healthy lifestyle}

Promotion of a healthy lifestyle is essential for AYA cancer patients. Oncologists may play a central role in supporting health-promoting behaviors by encouraging good nutrition and exercise and laying the ground work for lifelong followup care to screen for second cancers and late effects. AYAs should be assessed for and counseled about health-harming behaviors such as substance use and risky sexual activity during treatment. Predictors of health-harming behavior have been investigated, and patients who have issues with mental health or cognition tend to be at increased risk. In addition, patients who interact infrequently with medical professionals may have increased rates of health-harming behaviors. ${ }^{6}$

AYA patients should be educated about health promotion at all appointments, thus highlighting the need for adherence to scheduled follow-up. On-and-off therapy encounters with healthcare professionals present opportunities to provide individualized counseling to promote healthy behaviors. Additional education or interventions targeted at AYA patients and survivors may also be undertaken by oncology programs that are able to focus on AYA care, paying particular attention to differences in age, sex, and learning style.

\section{Growth and development}

Linear growth, final adult stature, and pubertal development may all be affected in patients who are diagnosed with cancer during adolescence and young adulthood. Causes of impaired growth and pubertal development include growth hormone deficiency, primary thyroid dysfunction, premature sexual maturation, hypogonadism, ovarian failure, and inadequate nutrition. ${ }^{7}$ The most common endocrinopathies in cancer patients and survivors are hypothyroidism and growth hormone deficiency secondary to radiation therapy. ${ }^{8}$ AYA patients should have height, weight, and sexual maturation monitored with each visit and should be screened for hormonal deficiencies as clinically indicated. Nutrition must also be considered in AYAs because adequate nutrition is necessary for both appropriate growth and development and in combating obesity.

\section{Symptom management}

Symptom management may play a large role in AYA quality of life. Studying the symptom burden during cancer therapy has gained momentum in the past several decades, although interventions to address symptom management have not kept pace with current advances in cancer therapy. ${ }^{9}$ Symptoms may include fatigue, nausea, pain, lack of appetite, and psychological distress. Support of symptom management has the potential to make significant contributions to the care of the AYA patient.

There is still need for further investigation into optimal timing and mechanisms for health counseling to promote growth, development, and symptom management among AYA patients. Healthcare providers should seek to maximize health-related education to promote health-protective behaviors and decrease symptom burden at each patient encounter and through use of a multidisciplinary team.

\section{Sexual and reproductive health}

AYAs in the general population undergo physical maturation, develop their sexual identity, and work to define romantic relationships. Treatment for cancer during adolescence and young adulthood may halt or delay both the physical changes and social milestones that occur during adolescence. AYA survivors cite sexuality and reproductive health as one of the most important issues facing them; however, these issues are not often acknowledged as part of routine oncologic care. ${ }^{10}$ Therefore, issues related to current and future fertility and general sexual health should be addressed and frequently revisited with AYAs as they progress through treatment and transition to survivorship.

Sexuality may encompass or refer to sexual identity, sexual development, sexual relationships, sexual function, and body image, all of which may be affected by cancer treatment. Although AYAs might have an initial shift in focus with a cancer diagnosis, they are still individuals with emerging sexual identities and practices. There are data to suggest that 
adolescents with chronic illness engage in sexual activity in equal rates to their healthy peers, making conversations surrounding sexual health a necessity for these groups. ${ }^{11}$

Although conversations surrounding sexual health are difficult to initiate for both patient and provider, even in those without cancer, providers who lack the initiative to initiate honest and frank conversations are missing an opportunity to ensure appropriate education and expectations in a vulnerable population. ${ }^{12}$ Lack of attention to sexual health issues by medical providers, accompanied by traditional AYA risk-taking behaviors, could lead to sexually transmitted infections (STIs) or unintended pregnancy. Both STIs and unintended pregnancy and their sequelae may affect future fertility and affect health outcomes. ${ }^{13}$

Sexuality and sexual function may also be directly affected by cancer treatment. In younger adolescents still undergoing puberty, pubertal problems may develop because of treatment effect on the central nervous system, the hypothalamic-pituitary axis, or the gonads directly. ${ }^{14}$ In addition, patients may have a variety of concerns about body image because of hair loss, weight gain or loss, the presence of implantable devices, and surgical scars. ${ }^{15}$ Problems of sexual function are also noted by cancer patients and survivors, although oftentimes they are not addressed during follow-up care. ${ }^{16}$

For all AYA cancer patients, open conversations about sexual health, including sexual practices, function, and body image concerns, should be elicited by providers and readdressed throughout treatment and survivorship. No assumptions should be made about sexual orientation, practices, or knowledge of the patient to ensure adequate education and support are provided.

\section{Fertility}

It is well known that radiation therapy and certain chemotherapeutic agents may cause infertility and/or gonadal failure. ${ }^{17}$ However, in many cases, fertility may be unaffected. In this way, fertility needs to be addressed in two often contradictory formats. First, AYAs must be offered appropriate information about their current fertility and appropriate contraception during treatment to prevent unintended pregnancy. Simultaneously, AYAs must be counseled on potential infertility as a result of cancer treatment and offered appropriate support and fertility preservation options.

Many cytotoxic drugs, as well as radiotherapy, are teratogenic, and conception during chemotherapy may result in abortion of the embryo or in congenital abnormalities, emphasizing the need for effective contraception in sexually active young people undergoing treatment for cancer. Often there is uncertainty on the part of the oncology provider about what methods are acceptable, but multiple professional organizations such as the World Health Organization, Centers for Disease Control and Prevention, and American College of Obstetrics and Gynecology have published medical eligibility criteria to assist practitioners in recommending and prescribing appropriate contraception for women with chronic illness. ${ }^{18}$ Ideally, to protect these patients against pregnancy and STIs, dual protection would be the best option; that is, condom plus another method with a lower failure rate for pregnancy. ${ }^{18,19}$

Infertility is a quality-of-life issue for cancer survivors and their families. In recent years, numerous treatment options have become available for patients at risk for infertility; thus, the American Society of Clinical Oncology recommends addressing possible infertility, with patients being treated during reproductive years. ${ }^{20}$ Counseling about infertility should be completed at the start of treatment so that fertility preservation options can be maximized. ${ }^{21}$

For males, testicular dysfunction is one of the most common adverse effects of cancer therapy. Males treated for pediatric cancers and followed into adulthood are less likely to sire a pregnancy than sibling controls when exposed to high doses of radiation and/or alkylating agents. ${ }^{22}$ Thus, cryopreservation of sperm is now standard practice. Early identification and prompt referral for sperm banking are key factors in helping male adolescents with cancer and should be offered regardless of prognosis. ${ }^{23}$

Women have a standard number of follicles at birth, and cancer therapy accelerates the natural decline in follicles. This effect of therapy may be more significant for an older adolescent or young adult than for a pediatric patient, significantly altering reproductive potential by putting them at risk for premature ovarian failure. ${ }^{24}$ Following pediatric cancer survivors into adulthood, lower rates of pregnancy are observed compared with siblings for those who received hypothalamic or gonadal radiation or who received high doses of alkylating agents. ${ }^{25}$ Embryo preservation is the most wellestablished option for fertility preservation, but for an AYA patient who may not be willing to use donor sperm, oocyte preservation is an alternative. ${ }^{26}$

The 2010 AYA LIVESTRONG survey found that about one-quarter of AYAs took steps to preserve their fertility before their cancer treatment began, and this number may have been higher if lack of knowledge, cost, and time could be combated. ${ }^{27}$ Recommendations for discussions about fertility and fertility preservation include discussing 
fertility at the time of diagnosis and having an established referral mechanism for fertility preservation and long-term follow-up. ${ }^{28}$ In addition, when available, a dedicated fertility team is preferable to the primary oncologist for conversations about fertility preservation. ${ }^{25}$

\section{Mental health}

Chronic illness has been shown to increase depressed mood and need for psychological intervention in AYA patients. ${ }^{29}$ AYAs with cancer experience multiple stressors, which may lead to feelings of depression or anxiety, including fears of death, recurrence, or other late effects. AYAs must also cope with their own emotions about social interactions and sometimes manage the emotions of their friends and family members. Thus, the psychosocial and supportive care needs of AYAs with cancer tend to be broader in scope and intensity than such needs in younger and older patients because of the many emotional, developmental, and social changes and transitions that occur during this stage of life. ${ }^{30}$

Because adolescents and young adults are more vulnerable to stress, ideally, all AYA cancer patients should be offered psychosocial support throughout treatment. ${ }^{31}$ Unfortunately, in the AYA HOPE Study, 15\% of AYA cancer survivors reported an unmet need for mental health support and 14\% reported an unmet need for support groups. This lack of psychosocial support has been associated with worse overall quality of life. ${ }^{32}$

Cancer survivors struggle with issues of mental health as well. Zelter et al reported outcomes of childhood cancer survivors and found they had more global distress and poorer scores on physical domains of health-related quality-of-life measures compared with their siblings. Langeveld et al found that long-term survivors worried about their fertility, getting/changing jobs, and obtaining insurance. Female sex, unemployment, severe health problems, and low self esteem were predictors of worse quality of life in survivors. ${ }^{33}$ Health-related hindrance of personal goals may affect AYA patients during a time when personal goals are a central part of development and transition to adulthood. ${ }^{34}$ Psychological adjustment may also take the form of posttraumatic stress disorders. Taylor et al found a local prevalence of posttraumatic stress symptoms and posttraumatic stress disorder (up to $14 \%$ of survivors studies) that was more prevalent in females and those with late effects, highlighting the need for services for these survivors. ${ }^{35}$

Issues with social anxiety affecting quality of life may actually increase with time since diagnosis and treatment, suggesting psychosocial adjustment and support must be continually addressed during treatment and well into survivorship. ${ }^{36}$ Current trends in research support the need for a stress-coping model framework and developmentally appropriate interventions in which psychosocial issues are addressed in the context of AYA maturation and patient cognition. ${ }^{30}$

\section{Health behaviors}

Prevalence rates of substance use are alarmingly high among adolescents and young adults, with between $8.5 \%$ and $25 \%$ reporting cigarette smoking, binge drinking, illicit drug use, and/or marijuana use in the past 30 days. ${ }^{37}$ AYA with cancer are especially vulnerable to health risks from substance use, as substances can exacerbate hepatotoxicity, pulmonary toxicity, or cardiac toxicity that may result from chemotherapy or radiation. Substance use may also adversely affect mood, adherence, or sensitivity to medications. ${ }^{38}$

AYAs with cancer, as well as survivors, have been studied to assess their risk for substance use as compared with the general population. Tyc et al and Carpentier et al examined adolescents receiving active treatment and found about 5\% of them endorsed using substances, which was a lower percentage than for healthy peers. ${ }^{39,40}$

The underlying reasons for substance use are multifactorial in the general population, including experimentation, fun, or coping. Peer influence has also been shown to be a prominent determinate of substance use in adolescence. There may be certain demographic and treatment-related characteristics of AYAs with cancer that place them at increased risk for substance use. Carpentier et al found that including more time since diagnosis and higher adolescentrated quality of life may be protective against health-harming behaviors such as substance use. ${ }^{40}$ There may also be some aspects of coping in cancer patients who choose to use substances. ${ }^{41}$ Therefore, although health-risk behavior such as substance use should be discussed with all AYA patients by providers, there may be subsets of patients to target for intervention.

AYA patients should receive counseling about substance use in a nonjudgmental fashion. The medical risks of use, including exacerbation of organ toxicity, drug-drug interactions, and potential for increasing risk of second cancers, should be highlighted. If AYAs disclose use, appropriate counseling and treatment services should be offered to them.

\section{Social issues}

AYA oncology patients must navigate schooling and employment, acquiring insurance, and establishing and maintaining 
stable relationships with family and friends. They may also be looking ahead and be considering their ability to marry or have children as a cancer survivor. Thus, relating with family, peers, and health professionals becomes an important aspect of life for AYAs with cancer.

\section{Education and employment}

Continuation or reintegration into social systems such as school may be difficult for AYA patients. Educational issues include repeating time in school or requiring extra assistance or tutoring. In some cases, even those who completed degrees finished with a delay.

Employment may be difficult to obtain or maintain during treatment or as a survivor. Patients may have lost opportunities or are underemployed because of their diagnosis or subsequent late effects. ${ }^{42}$ In addition, many AYA survivors may have limited job choice or job mobility because of their need to maintain appropriate health insurance or closeness to a particular geographic region secondary to care. The Childhood Cancer Survivorship Study has published data to suggest that unemployment among cancer survivors is directly related to their health, and unemployed cancer survivors reported poorer levels of physical functioning than employed survivors. ${ }^{43}$

\section{Relationships}

Establishing and maintaining peer and romantic relationships is one of the most important developmental milestones in adolescence and young adulthood. However, it is not uncommon for AYA cancer patients to experience disruptions in friendships, family life, and romantic relationships or to experience social isolation resulting from large periods of time spent away from home, school, or work during treatment. In addition, the very experience of a cancer diagnosis can alter identity and sense of self, complicating relationships and navigation of social systems for AYA patients. ${ }^{44}$ Initial treatment intensity, time since diagnosis, and severity of late effects after therapy may be associated with some indices of social adjustment. ${ }^{45}$

\section{The role of family}

Parents, spouses, or other family support of AYA with cancer often have an important role in care and support of the AYA patient, and it is imperative that providers take this into consideration. ${ }^{46}$ Parents of AYAs may fall into many roles in relation to both AYAs and physician relationships, including more active and collaborative roles or more passive roles; therefore, it is critical for healthcare providers to consider the role that caregivers intend to play and to adapt accordingly. This will ensure the most effective approach in treating the diagnosed patient and will establish common ground among all parties involved. ${ }^{47}$

AYA patients may also be parents themselves. In this case, AYAs may feel as though the disease interrupts the continuity of care they can give to their own children. Their adjustment becomes more complex, as they must cope with treatment themselves and also provide support for their children while striving to be a good parent. ${ }^{48} \mathrm{~A}$ cancer diagnosis may affect finances, social and mental aspects of current family life, and the expected future a parent has envisioned for their family, leading to a sense of guilt or helplessness concerning their role as a parent. ${ }^{49}$

AYAs undergoing cancer therapy should be counseled as to the difficulties of and mechanisms for support for continuation and reintegration into social systems, including education, employment, and peer and family relationships. When possible, survivors should be offered assistance with resources for aid with educational achievement and job training. AYAs should also be encouraged and supported to maintain relationships during and after therapy.

\section{Transition to adulthood and survivorship care}

One of the first issues that must be discussed at diagnosis for any AYA patient is whether they will be treated in a pediatric or adult institution. This decision must be made according to the medical and psychosocial needs of the patient, although it should be noted that both pediatric and adult institutions may lack support services or specialists who focus on AYA care. ${ }^{50}$ As this decision is being made, consultation of a pediatric oncology team by an adult team, or vice versa, may be useful, and difficult decisions about reasons to transition care between institutions may need to be discussed at diagnosis or during treatment.

The need for transition to appropriate survivorship care is also critical when screening patients for late effects of cancer diagnosis and treatment. The Childhood Cancer Survivorship Study followed pediatric survivors into adulthood and found that almost half of cancer survivors experience a serious or life-threatening condition between 5 and 30 years after diagnosis, and almost three-quarters develop at least one chronic condition. ${ }^{51}$ These late effects include, but are not limited to, cardiac, pulmonary, and endocrine diagnoses, as well as secondary malignancies. In addition, survivors require lifelong care that focuses not only on the medical risks arising from their cancer therapy but also on 
the psychosocial, educational, and vocational implications of surviving cancer. ${ }^{4}$

Despite these risks, the majority of survivors are not receiving the cancer-related follow-up care that is recommended. ${ }^{52}$ The correct timing of transition to survivorship care and correct survivorship team or provider and model of care is often difficult for both patients and providers to identify. In addition, providers who assume care of survivors often lack the knowledge needed to care for cancer survivors and ensure appropriate screening and follow-up. ${ }^{53}$

Characteristics of AYAs, their parents, and providers play a critical role in successful transition. The Childhood Cancer Survivorship Study identified predictors of lack of follow-up care as survivors, including older age, longer time from diagnosis, male sex, lack of health insurance, and lack of concern for future health. ${ }^{6}$ Survivors with physical symptoms such as pain or increased anxiety have been shown to have higher rates of follow-up care. ${ }^{54}$ Granek et al found that psychosocial variables such as identification with being a survivor and fear and anxiety could act as both a motivator for and a hindrance to successful transition. ${ }^{55}$

Adolescents and young adults should be counseled on medically and developmentally appropriate transitions during and at the completion of therapy. Factors for successful transition also need to be factored into transition planning and transition readiness assessments. ${ }^{34}$ Services that may be helpful to AYAs throughout treatment should be provided throughout the transition to survivorship and adult-focused care, such as education, appropriate surveillance and screening, management of cancer-related complications, and psychosocial support. ${ }^{4}$

\section{Conclusion}

A cancer diagnosis during adolescence or young adulthood disrupts a time of social and developmental transitions. Young people undergoing cancer treatment and in transition to survivorship care should be supported in engaging in developmentally normal tasks and milestones. Unfortunately, research suggests that AYA patients are inadequately served by currently available services, which directly affects the health-related quality of life of many AYAs diagnosed with cancer. ${ }^{32}$

Healthcare teams caring for AYA patients have an opportunity to influence behavioral practices by assessing quality of life during care and addressing factors that enhance the AYA's sense of vulnerability to health problems while providing personalized health counseling that encourages the practice of health-promoting behavior. Where possible, AYA patients should be treated by an expert in their disease process and an expert in their age group. Dedicated AYA clinical teams are likely the most proficient in treating both the medical and psychosocial needs of this group. Where specific clinical AYA teams are not available, individual providers should try to address physical health, mental health, and social issues that affect AYA patients and offer appropriate age-based support services to optimize healthrelated quality of life.

\section{Disclosure}

The authors report no conflicts of interest in this work.

\section{References}

1. National Cancer Institute. Adolescents and young adults with cancer. Bethesda, MD: National Cancer Institute. Available from: http://www. cancer.gov/cancertopics/aya. Accessed July 7, 2014.

2. National Institute for Clinical Excellence. Improving Outcomes Guidance for Children and Young People with Cancer. London, UK: National Institute for Clinical Excellence; 2005. Available from: http:// www.nice.org.uk/guidance/csgcyp/resources/improving-outcomes-inchildren-and-young-people-with-cancer-manual-update-2. Accessed July 7, 2014.

3. Smith AW, Bellizzi KM, Keegan TH, et al. Health-related quality of life of adolescent and young adult patients with cancer in the United States: the Adolescent and Young Adult Health Outcomes and Patient Experience study. J Clin Oncol. 2013;31(17):2136-2145.

4. Nathan PC, Hayes-Lattin B, Sisler JJ, Hudson MM. Critical issues in transition and survivorship for adolescents and young adults with cancers. Cancer. 2011;117(10)(Suppl):2335-2341.

5. Nass S, Patlak M, Patlack R; National Cancer Policy Forum, Board on Health Care Services. Identifying and Addressing the Needs of Adolescents and Young Adults with Cancer: Workshop Summary. Washington, DC: The National Academies Press; 2013. Available from: http://books.nap. edu/openbook.php?record_id=18547. Accessed July 7, 2014.

6. Lee YL, Santacroce SJ, Sadler L. Predictors of healthy behaviour in long-term survivors of childhood cancer. J Clin Nurs. 2007;16(11C): 285-295.

7. Sklar CA. Growth and pubertal development in survivors of childhood cancer. Pediatrician. 1991;18(1):53-60.

8. Duffner PK. Long-term effects of radiation therapy on cognitive and endocrine function in children with leukemia and brain tumors. Neurologist. 2004;10(6):293-310.

9. Kestler SA, LoBiondo-Wood G. Review of symptom experiences in children and adolescents with cancer. Cancer Nurs. 2012;35(2): E31-E49.

10. Hordern AJ, Street AF. Communicating about patient sexuality and intimacy after cancer: mismatched expectations and unmet needs. Med J Aust. 2007;186(5):224-227.

11. Surís JC, Resnick MD, Cassuto N, Blum RW. Sexual behavior of adolescents with chronic disease and disability. J Adolesc Health. 1996;19(2):124-131.

12. Tao G, Irwin KL, Kassler WJ. Missed opportunities to assess sexually transmitted diseases in US adults during routine medical checkups. Am J Prev Med. 2000;18(2):109-114.

13. Gottlieb SL, Newman LM, Amin A, Temmerman M, Broutet N. Sexually transmitted infections and women's sexual and reproductive health. Int J Gynaecol Obstet. 2013;123(3):183-184.

14. Shalet SM, Brennan BM. Puberty in children with cancer. Horm Res. 2002;57(Suppl 2):39-42.

15. Pendley JS, Dahlquist LM, Dreyer Z. Body image and psychosocial adjustment in adolescent cancer survivors. J Pediatr Psychol. 1997;22(1):29-43. 
16. Greaves P, Sarker SJ, Chowdhury K, et al. Fertility and sexual function in long-term survivors of haematological malignancy: using patientreported outcome measures to assess a neglected area of need in the late effects clinic. Br J Haematol. 2014;164(4):526-535.

17. Ginsberg JP. Educational paper: the effect of cancer therapy on fertility, the assessment of fertility and fertility preservation options for pediatric patients. Eur J Pediatr. 2011;170(6):703-708.

18. American College of Obstetricians and Gynecologists (ACOG). Use of hormonal contraception in women with coexisting medical conditions. ACOG Practice Bulletin no 73. Washington, DC: American College of Obstetricians and Gynecologists; 2006. Available from: http://www. guideline.gov/content.aspx?id=10924. Accessed August 8, 2014.

19. Laurence V, Gbolade BA, Morgan SJ, Glaser A. Contraception for teenagers and young adults with cancer. Eur J Cancer. 2004;40(18): 2705-2716.

20. Lee SJ, Schover LR, Partridge AH, et al; American Society of Clinical Oncology. American Society of Clinical Oncology recommendations on fertility preservation in cancer patients. J Clin Oncol. 2006;24(18): 2917-2931.

21. Ginsberg JP. New advances in fertility preservation for pediatric cancer patients. Curr Opin Pediatr. 2011;23(1):9-13.

22. Green DM, Kawashima T, Stovall M, et al. Fertility of male survivors of childhood cancer: a report from the Childhood Cancer Survivor Study. J Clin Oncol. 2010;28(2):332-339.

23. Hobbie WL, Ogle SK, Ginsberg JP. Fertility concerns for young males undergoing cancer therapy. Semin Oncol Nurs. 2009;25(4):245-250.

24. Fallat ME, Hutter J, American Academy of Pediatrics Committee on Bioethics; American Academy of Pediatrics Section on Hematology/ Oncology, American Academy of Pediatrics Section on Surgery. Preservation of fertility in pediatric and adolescent patients with cancer Pediatrics. 2008; 121(5):1461-1469.

25. Green DM, Kawashima T, Stovall M, et al. Fertility of female survivors of childhood cancer: a report from the childhood cancer survivor study. J Clin Oncol. 2009;27(16):2677-2685.

26. Levine J, Canada A, Stern CJ. Fertility preservation in adolescents and young adults with cancer. J Clin Oncol. 2010;28(32):4831-4841.

27. "I Learned Enough to Live with It" Is Not Good Enough: Challenges Reported By Post Treatment Cancer Survivors in the LIVESTRONG Surveys. A Livestrong Report. Austin, TX: Livestrong; 2011. Available from: http://images.livestrong.org/downloads/flatfiles/what-we-do/ourapproach/reports/challenges/LSSurvivorSurveyReport_final.pdf?_ga= 1.123287426.807642754.1413604037. Accessed August 20, 2014.

28. Metzger ML, Meacham LR, Patterson B, et al. Female reproductive health after childhood, adolescent, and young adult cancers: guidelines for the assessment and management of female reproductive complications. J Clin Oncol. 2013;31(9):1239-1247.

29. Surís JC, Parera N, Puig C. Chronic illness and emotional distress in adolescence. J Adolesc Health. 1996;19(2):153-156.

30. Adolescent and Young Adult Oncology Progress Review Group. Closing the Gap: Research and Care Imperatives for Adolescents and Young Adults with Cancer. Report of the Adolescent and Young Adult Oncology Progress Review Group. Bethesda, MD: Adolescent and Young Adult Oncology Progress Review Group. Available from: http://deainfo.nci.nih.gov/advisory/ ncab/139_0906/presentations/AYAO.pdf. Accessed January 31, 2014.

31. Hobbie WL, Stuber M, Meeske K, et al. Symptoms of posttraumatic stress in young adult survivors of childhood cancer. J Clin Oncol. 2000;18(24):4060-4066.

32. Smith AW, Parsons HM, Kent EE, et al; AYA HOPE Study Collaborative Group. Unmet Support Service Needs and Health-Related Quality of Life among Adolescents and Young Adults with Cancer: The AYA HOPE Study. Front Oncol. 8, 2013;3:75.

33. Langeveld NE, Grootenhuis MA, Voûte PA, de Haan RJ, van den Bos C. Quality of life, self-esteem and worries in young adult survivors of childhood cancer. Psychooncology. 2004;13(12):867-881.

34. Schwartz LA, Drotar D. Health-related hindrance of personal goal pursuit and well-being of young adults with cystic fibrosis, pediatric cancer survivors, and peers without a history of chronic illness. J Pediatr Psychol. 2009;34(9):954-965.
35. Taylor N, Absolom K, Snowden J, Eiser C; Late Effects Group Sheffield. Need for psychological follow-up among young adult survivors of childhood cancer. Eur J Cancer Care (Engl). 2012;21(1):52-58.

36. Schultz KA, Ness KK, Whitton J, et al. Behavioral and social outcomes in adolescent survivors of childhood cancer: a report from the childhood cancer survivor study. J Clin Oncol. 2007;25(24):3649-3656.

37. Johnston L, O'Malley PM, Bachman JG, Schulenberg JE. Monitoring the Future National Results on Adolescent Drug Use: Overview of Key Findings, 2011. Ann Arbor: Institute for Social Research, The University of Michigan; 2012.

38. Palmer S, Thomas D. A Practice Framework for Working with 15-25 Year-Old Cancer Patients Treated Within the Adult Health Sector. Melbourne,Australia: OnTrac@PeterMac Adolescent and Young Adult Cancer Service; 2008. Available from: http:/www1.petermac.org/ ontrac/pdf/AYA-Practice-Framework.pdf. Accessed July 7,2014.

39. Tyc VL, Lensing S, Klosky J, Rai SN, Robinson L. A comparison of tobacco-related risk factors between adolescents with and without cancer. J Pediatr Psychol. 2005;30(4):359-370.

40. Carpentier MY, Mullins LL, Elkin TD, Wolfe-Christensen C. Predictors of health-harming and health-protective behaviors in adolescents with cancer. Pediatr Blood Cancer. 2008;51(4):525-530.

41. Szalda DE, Brumley LD, Danielson CK, Schwartz LA. Exploratory Analyses of Substance use in adolescents with and without cancer. J Adolesc Young Adult Oncol. 2013;2(2):77-82.

42. de Boer AG, Verbeek JH, van Dijk FJ. Adult survivors of childhood cancer and unemployment: A metaanalysis. Cancer. 2006;107(1):1-11.

43. Kirchhoff AC, Leisenring W, Krull KR, et al. Unemployment among adult survivors of childhood cancer: a report from the childhood cancer survivor study. Med Care. 2010;48(11):1015-1025.

44. Mathieson CM, Stam HJ. Renegotiating identity: cancer narratives Sociol Health Illn. 1995;17(3):283-306.

45. Zebrack BJ. Cancer survivor identity and quality of life. Cancer Pract. 2000;8(5):238-242.

46. Pyke-Grimm KA, Degner L, Small A, Mueller B. Preferences for participation in treatment decision making and information needs of parents of children with cancer: a pilot study. J Pediatr Oncol Nurs. 1999;16(1):13-24.

47. Mack JW, Wolfe J, Grier HE, Cleary PD, Weeks JC. Communication about prognosis between parents and physicians of children with cancer: parent preferences and the impact of prognostic information. J Clin Oncol. 2006;24(33):5265-5270.

48. ElmbergerE, Bolund C, Lützén K. Experience of dealing with moral responsibility as a mother with cancer. Nurs Ethics. 2005;12(3):253-262.

49. Elmberger E, Bolund C, Lützén K, Eva Elmberger, Christina Bolund, Ki. Transforming the exhausting to energizing process of being a good parent in the face of cancer. Health Care Women Int. 2000;21(6): 485-499.

50. Zebrack B, Mathews-Bradshaw B, Siegel S; LIVESTRONG Young Adult Alliance. Quality cancer care for adolescents and young adults: a position statement. J Clin Oncol. 2010;28(32):4862-4867.

51. Oeffinger KC, Mertens AC, Sklar CA, et al; Childhood Cancer Survivor Study. Chronic health conditions in adult survivors of childhood cancer. N Engl J Med. 2006;355(15):1572-1582.

52. Nathan PC, Greenberg ML, Ness KK, et al. Medical care in long-term survivors of childhood cancer: a report from the childhood cancer survivor study. J Clin Oncol. 2008;26(27):4401-4409.

53. Suh E, Daugherty CK, Wroblewski K, et al. General internists' preferences and knowledge about the care of adult survivors of childhood cancer: a cross-sectional survey. Ann Intern Med. 2014;160(1):11-17.

54. Nathan PC, Ford JS, Henderson TO, et al. Health behaviors, medical care, and interventions to promote healthy living in the Childhood Cancer Survivor Study cohort. J Clin Oncol. 2009;27(14):2363-2373.

55. Granek L, Nathan PC, Rosenberg-Yunger ZR, et al. Psychological factors impacting transition from paediatric to adult care by childhood cancer survivors. J Cancer Surviv. 2012;6(3):260-269. 


\section{Publish your work in this journal}

Clinical Oncology in Adolescents and Young Adults is an international, peer-reviewed, open access journal publishing original research, reports, editorials, reviews and commentaries on all aspects of epidemiology, diagnosis and treatment of cancers in adolescents and young adults. The manuscript management system is completely

Submit your manuscript here: http://www.dovepress.com/clinical-oncology-in-adolescents-and-young-adults-journal online and includes a very quick and fair peer-review system. Visit http://www.dovepress.com/testimonials.php to read real quotes from published authors. 